\title{
Survival After Emergency General Surgery: What can We Learn from Enhanced Recovery Programmes?
}

\author{
N. Quiney ${ }^{1,2}$ - G. Aggarwal ${ }^{1,2} \cdot$ M. Scott ${ }^{1,2} \cdot$ M. Dickinson ${ }^{1,2}$
}

Published online: 26 January 2016

(C) Société Internationale de Chirurgie 2016

\begin{abstract}
Enhanced recovery after surgery (ERAS) has been adopted by many centres and across whole healthcare systems. The results have shown significant reductions in length of stay and postoperative complications. However, there has been very little change in these factors and mortality in emergency surgery. Can we learn from principles of ERAS for emergency abdominal surgery?
\end{abstract}

Over the last 15 years, there has been a quiet revolution in the delivery of elective surgical care. The main drivers of these improvements are a combination of improved surgical techniques (laparoscopic and robotic assisted) and evidence-based perioperative pathways, otherwise termed enhanced recovery after surgery (ERAS) [1]. The results have seen significant reductions in postoperative complications, length of stay (LOS) and an improvement in longterm survival after some cancer operations [2, 3].

In stark contrast, there has been little improvement in outcomes or morbidity for patients undergoing emergency abdominal surgery, despite evidence outlining the high morbidity and mortality associated with this cohort.

In 1996, Pearse et al. examined 4.1 million surgical procedures in the UK's Intensive Care National Audit and

N. Quiney

nialquiney@nhs.net

G. Aggarwal

geetaaggarwal@nhs.net

1 Department of Anaesthesia and Perioperative Medicine, Royal Surrey County Hospital NHS Foundation Trust, Egerton Road, Guildford, Surrey GU2 7XX, UK

2 Surrey Perioperative Anesthesia Critical Care Research Group (SPACeR), University of Surrey, Guildford GU2 7XH, UK
Research Centre database [4]. Pearse identified a subset of approximately 500,000 patients who made up only $12.5 \%$ of the study population but accounted for $83.8 \%$ of all observed 30-day mortality. Many of these patients were elderly with pre-existing comorbidities undergoing emergency abdominal surgery.

In 2012, two further publications from the US and UK identified high mortality rates in patients undergoing emergency general surgical operations. Al Temimi et al. [5] retrospectively identified 37,553 patients who had undergone emergency laparotomy from the American College of Surgeons National Surgical Quality Improvement Program database (2005-2009) in the US. They found a 30-day mortality rate of $14 \%$. In the UK, the NHS Emergency Laparotomy Network prospectively studied 1853 patients from 37 hospitals and found a similar 30-day mortality of $14.9 \%$ [6].

In the UK, it has been estimated that an emergency laparotomy is carried out each year for every 1100 patients [7]. This would approximate to 55,000 operations carried out per year, and based on $14.6 \%$ mortality, potentially 8000 deaths.

It has become clear that emergency general surgical operations carry a mortality rate at least ten times higher than many similar elective procedures.

In Denmark, Møller et al. [8] reported the effect of introducing a standardised care pathway for patients 
undergoing emergency surgery for peptic ulcer perforation. They showed a $37 \%$ reduction in in-hospital mortality rate compared to both historical and concurrent national control groups [8]. A more recent study by Huddart et al. [9] demonstrated a significant reduction in mortality for patients undergoing emergency laparotomy. This quality improvement project across four large hospitals in the UK applied the simple principles of evidence-based medical care and quality improvement methodology. By expediting resuscitation, assessment for sepsis and administration of broad-spectrum antibiotic therapy, definitive care with the use of goal-directed fluid resuscitation and routine admission to the intensive care unit, the four centres involved were able to reduce risk-adjusted 30-day mortality by $42 \%$.

What similarities do the methods used in these two publications in emergency surgery have with the principles of ERAS in elective surgery?

Surgical insult drives a physiological stress response, the magnitude of which is proportional to tissue damage and blood loss. The aim of the ERAS elements is to minimise this effect and improve the metabolic response to surgery.

The components of ERAS can be divided into preoperative, intraoperative and postoperative factors and most elements are well evidence based [10].

Preoperative counselling, assessment and optimisation.

Standards of care antibiotics, thromboprophylaxis, prevention of postoperative nausea and vomiting, maintenance of normothermia.

Elements to reduce the pathophysiologic insult avoidance of bowel preparation, avoidance of nasogastric tubes, minimally invasive surgery, no drains, early removal of catheters.

Elements to avoid postoperative gut dysfunction and ileus avoidance of salt and water overload, stimulation of gut motility, non-opioid oral analgesia, non-steroidal antiinflammatory drugs.

Elements to improve the metabolic response to surgery avoidance of prolonged starvation, carbohydrate loading and early enteral feeding.

Audit compliance and outcome.

The key to enhanced recovery programmes of all types is to:

(1) Identify what evidence is available that might contribute to improved outcomes

(2) Include this evidence into a time-based pathway

(3) Set up hospital systems to ensure that the elements of these pathways are consistently implemented and complied with.

However, unlike ERAS pathways, there is not the breadth of good quality evidence relating to patients undergoing emergency surgery. It is worthy to identify the clinical issues for patients undergoing emergency surgery that are not easily amenable to ERAS principles.

- Delay in diagnosis Unlike patients presenting for elective surgery, patients requiring emergency laparotomy often present to the Emergency Department, occasionally with non-specific symptoms and may end up under a different, altogether non-surgical speciality before the diagnosis is finally made.

- Delay in resuscitation Many patients present with gut dysfunction such as ischaemic bowel or with intraabdominal soiling causing sepsis. These patients are essentially presenting with a complication before surgery [11]. The failure to expedite surgical intervention to drain and clear sources of infection in patients who require emergency laparotomy is well described [12].

- Delay in antibiotics Severe sepsis is poorly recognised and treated [13] and further increases the risk of death. The mortality for septic patients increases by $7 \%$ by every hour that antibiotic administration is delayed [12]. Increasing compliance with the resuscitation bundle of the Surviving Sepsis Campaign has been shown to effectively reduce mortality in patients with septic shock [14].

- Delay in definitive care Patients are often dehydrated secondary to bowel obstruction, reduced oral intake and a systemic inflammatory response related to sepsis. Hypovolaemia, insulin resistance and decreased glycogen reserves may well all be present [15]. Too often the risk of death is not appreciated for patients undergoing emergency general surgery. The fluid shifts associated with these pathophysiological insults are also often poorly recognised. The use of individualised goaldirected fluid resuscitation has been shown to reduce postoperative complications in high-risk patients [16].

- Inadequate postoperative care Postoperative patients are often managed in areas in the hospital other than intensive care. The development of a postoperative complication significantly increases mortality risk both in the short and long term [17] Patients not admitted to ICU after complex surgery who develop complications have a higher mortality rate compared to patients who are admitted directly after surgery to the intensive care unit [18].

Although all these aspects of care mitigate the use of some ERAS principles, the use of an evidence-based care bundle by Huddart et al. [9] shows that the consistent delivery of evidence-based medicine can significantly reduce mortality for patients undergoing emergency laparotomy (Fig. 1).

Once the best available evidence has been agreed by clinicians and assembled into a care pathway, the final area 


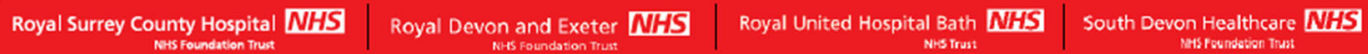 Emergency Laparotomy? एकी Emergency Laparotomy Pathway Quality Improvement Care-Bundle}

ALL PATIENTS presenting with emergency abdominal conditions THAT MAY REQUIRE EMERGENCY LAPAROTOMY are to be started on the Emergency Laparotomy ER Pathway and comply to the care-bundles goals below.

\section{Early Assessment and Resuscitation}

- MEWS within $\mathbf{3 0}$ minutes of arrival in hospital

- Outreach review if MEWS $>3$

- MRCS surgical review within $\mathbf{3 0}$ minutes of referral

- Measure arterial lactate

- Prompt fluid resuscitation

\section{E) Early Antibiotics}

- Within 1 hour if there is evidence of SIRS/sepsis

- Within 3 hours if there is suspicion of intra-peritoneal soiling

\section{Prompt diagnosis and Early surgery}

- CT scan - 'Code Emergency Laparotomy' prompts:

- 'Next Slot' prioritisation, scan within 2 hours of booking, verbal report within 1 hour of scan

- 'Next Slot' prioritisation on Emergency Theatre List

- Knife-to-skin within 6 hours of decision to operate

- Consultant surgeon and anaesthetist present in theatre

\section{Goal Directed Fluid Therapy}

- Goal Directed Fluid Therapy using cardiac output monitoring intra-operatively and for 6 hours post-operatively

\section{Post-operative Intensive Care for all}

- All patients to be cared for on intensive care

- If no intensive care bed is available - alternative level 2 area (e.g. Post Anaesthetic Care Unit or appropriately staffed recovery area, for at least 6 hours)

- Goal Directed Fluid Therapy for 6 hours post-operatively

Fig. 1 ELPQuiC care bundle. Huddart S et al. [9]. Use of a pathway quality improvement care bundle to improve outcomes after emergency laparotomy. (Permission for use granted by BJS) 
of focus should be on the reliable and consistent delivery of the care plan.

A stepwise approach is required to ensure that key goals of the pathway are consistently and reliably delivered. Quality improvement techniques are designed to ensure these goals. The 'Model for Improvement' is a framework for guiding improvement work focusing on identifying needs and using repeated Plan-Do-Study-Act cycles to achieve the original aims [19]. Run charts are a graphical display of performance over a period of time. Ideally LOS or ICU admission can be portrayed using run charts [19]. Driver diagrams and process mapping can be used to understand the workings of a complex system and therefore prompt change in areas of need [19].

Is it time for all professionals involved in the care of patients undergoing emergency laparotomy to start to examine their own practice and outcomes? A response to our current understanding of the high mortality rates associated with patients undergoing emergency laparotomy might come at three levels.

At a local level, health care providers must start to collect data on outcomes after emergency laparotomy (both 30- and 90-day mortality). These should be risk adjusted [20] using a tool such as P POSSUM or APACHE. The use of risk-adjusted cusum charts would be an ideal way of identifying current performance and variations, and allow benchmarking against other organisations or institutions.

All stakeholder groups in the provision of emergency surgery should focus their attention on improving quality of care for patients undergoing emergency laparotomy. Many such operations are carried out by surgeons from different subspecialties, e.g. colonic, hepatobiliary, or upper gastrointestinal subspecialties, therefore lack of ownership of these patients may be the reason there has been little focus in this area.

Further studies may also be proposed to further our understanding of the pathophysiology of emergency laparotomy. Novel ideas into on-going care following surgery, such as the use of physicians who specialise in this field should also be examined.

Finally, policy makers at a national level might consider how in their own unique healthcare system they might be able to bring about change in this area of surgical practice. Collection of data at a national level has been shown to be effective in driving improvement in healthcare delivery [21].

The levels of engagement for ERAS pathways have led to a significant improvement in delivery of care for patients undergoing many different elective surgical procedures.

It is time for organisations and healthcare professionals to apply the same standards of delivery to improve outcomes in emergency surgery as it has achieved in elective surgery.
Disclosure NF Quiney received an honoraria from Lidco plc for delivering educational talks about "emergency laparotomy". In addition, Lidcp plc assisted with travel costs.

\section{References}

1. Gustaffson UO, Scott MJ, Schwenk W (2013) Guidelines for perioperative care in elective colonic surgery: enhanced recovery after surgery (ERAS) society recommendations. World J Surg 37(2):259-284. doi:10.1007/s00268-012-1772-0

2. ERAS compliance group (2015) The impact of enhanced recovery protocol compliance on elective colorectal cancer resection: results from an international registry. Ann Surg 261:1153-1159

3. Vardhan KK, Neal KR, Dejong CHC et al (2010) The enhanced recovery after surgery (ERAS) pathway for patients undergoing major elective open colorectal surgery: a meta-analysis of randomized controlled trials. Clin Nutr 29(4):434-440

4. Pearse RM, Harrison DA, James P et al (2006) Identification and characterisation of the high risk surgical population in the United Kingdom. Crit Care 10(3):R81

5. Al Temimi MH, Griffee M, Enniss TM et al (2012) When is death inevitable after emergency laparotomy? Analysis of the American College of Surgeons National Surgical Quality Improvement Program database. J Am Coll Surg 215(4):503-511

6. Saunders DI, Murray D, Pichel AC, UK Emergency Laparotomy Network et al (2012) Variations in mortality after emergency laparotomy: the first report of the UK Emergency Laparotomy Network. Br J Anaesth 109(3):368-375

7. Shapter SL, Paul MJ, White SM (2012) Incidence and estimated annual cost of emergency laparotomy in England: is there a major funding shortfall? Anaesthesia 67(5):474-478

8. Møller MH, Adamsen S, Thomsen RW et al (2011) Multicentre trial of a perioperative protocol to reduce mortality in patients with peptic ulcer perforation. Br J Surg 98:802-810

9. Huddart S, Peden CJ, Swart M et al (2015) Use of a pathway quality improvement care bundle to reduce mortality after emergency laparotomy. Br J Surg 102(1):57-66

10. Scott MJ, Miller TE (2015) Pathophysiology of major surgery and the role of enhanced recovery pathways and the anesthesiologist to improve outcomes. Anesthesiol Clin 33(1):79-91

11. Peden C, Scott MJ (2015) Anesthesia for emergency abdominal surgery. Anesthesiol Clin 33(1):209-221

12. Kumar A, Roberts D, Wood KE et al (2006) Duration of hypotension before initiation of effective antimicrobial therapy is the critical determinant of survival in human septic shock. Crit Care Med 34(6):1589-1596

13. UK Sepsis Trust (2014) [Internet]. Toolkit: AMU management of sepsis. http://sepsistrust.org/wpcontent/files_mf/1409313292 AMUExecSummary2014.pdf. Accessed 26 June 2015

14. Levy MM, Rhodes A, Phillips GS et al (2015) Surviving Sepsis Campaign: association between performance metrics and outcomes in a 7.5-year study. Crit Care Med 43(1):3-12

15. Baldini G, Fawcett WJ (2015) Anesthesia for colorectal surgery. Anesthesiol Clin 33(1):93-123

16. Cecconi M, Corredor C, Arulkumaran N et al (2013) Clinical review: goal-directed therapy - what is the evidence in surgical patients? The effect on different risk groups. Crit Care 17:209

17. Khuri SF, Henderson WG, DePalma RG et al (2005) Determinants of long-term survival after major surgery and the adverse effect of postoperative complications. Ann Surg 242(3):326-341

18. Symons NR, Moorthy K, Almoudaris AM et al (2013) Mortality in high-risk emergency general surgical admissions. Br J Surg 100(10):1318-1325 
19. Colvin JR, Peden CJ (2012) Raising the standard: a compendium of audit recipes for continuous quality improvement in anaesthesia, 3rd edn. http://www.rcoa.ac.uk/system/files/CSQ-ARB2012_0.pdf. Accessed 12 Sept 2015

20. Steiner SH, Cook RJ, Farewell VT et al (2000) Monitoring surgical performance using risk-adjusted cumulative sum charts. Biostatistics 1(4):441-452
21. Society for Cardiothoracic Surgeons of Great Britain and Ireland. [Internet]. http://bluebook.scts.org. Accessed 14 May 2015 